\title{
Multimodal Imaging Brain Markers in Early Adolescence Are Linked with a Physically Active Lifestyle
}

\author{
Piergiorgio Salvan, ${ }^{1}$ Thomas Wassenaar, ${ }^{1}$ Catherine Wheatley, ${ }^{1}$ Nicholas Beale, ${ }^{2}$ Michiel Cottaar, ${ }^{1}$ Daniel Papp, ${ }^{1}$ \\ Matteo Bastiani, ${ }^{1,3,4}$ Sean Fitzgibbon, ${ }^{1}$ Euguene Duff, ${ }^{1}$ Jesper Andersson, ${ }^{1}$ Anderson M. Winkler, ${ }^{5,6}$ \\ Gwenaëlle Douaud, ${ }^{1}$ Thomas E. Nichols, ${ }^{1,7,8}$ Stephen Smith, ${ }^{1}$ Helen Dawes, ${ }^{2}$ and Heidi Johansen-Berg ${ }^{1}$ \\ ${ }^{1}$ Wellcome Centre for Integrative Neuroimaging, FMRIB Centre, Nuffield Department of Clinical Neurosciences, University of Oxford, John Radcliffe Hospital, \\ Oxford, OX3 9DU, United Kingdom, ${ }^{2}$ Centre for Movement, Occupational and Rehabilitation Sciences, Oxford Brookes University, Oxford, OX3 0BP, United \\ Kingdom, ${ }^{3}$ Sir Peter Mansfield Imaging Centre, School of Medicine, University of Nottingham, Nottingham, NG7 2RD, United Kingdom, ${ }^{4}$ National Institute \\ for Health Research Biomedical Research Centre, University of Nottingham, Nottingham, NG7 2UH, United Kingdom, ${ }^{5}$ Emotion and Development Branch, \\ National Institute of Mental Health, National Institutes of Health, Bethesda, MD 20892-9663, Maryland, ${ }^{6}$ Department of Psychiatry, Yale University School of \\ Medicine, New Haven, CT 06511, Connecticut, ${ }^{7}$ Oxford Big Data Institute, Li Ka Shing Centre for Health Information and Discovery, Nuffield \\ Department of Population Health, University of Oxford, Oxford, OX3 7LF, United Kingdom, and ${ }^{8}$ Department of Statistics, University of Warwick, \\ Coventry, CV4 7AL, United Kingdom
}

The World Health Organization promotes physical exercise and a healthy lifestyle as means to improve youth development. However, relationships between physical lifestyle and human brain development are not fully understood. Here, we asked whether a human brain-physical latent mode of covariation underpins the relationship between physical activity, fitness, and physical health measures with multimodal neuroimaging markers. In 50 12-year old school pupils (26 females), we acquired multimodal whole-brain MRI, characterizing brain structure, microstructure, function, myelin content, and blood perfusion. We also acquired physical variables measuring objective fitness levels, $7 \mathrm{~d}$ physical activity, body mass index, heart rate, and blood pressure. Using canonical correlation analysis, we unravel a latent mode of brain-physical covariation, independent of demographics, school, or socioeconomic status. We show that MRI metrics with greater involvement in this mode also showed spatially extended patterns across the brain. Specifically, global patterns of greater gray matter perfusion, volume, cortical surface area, greater white matter extra-neurite density, and resting state networks activity covaried positively with measures reflecting a physically active phenotype (high fit, low sedentary individuals). Showing that a physically active lifestyle is linked with systems-level brain MRI metrics, these results suggest widespread associations relating to several biological processes. These results support the notion of close brain-body relationships and underline the importance of investigating modifiable lifestyle factors not only for physical health but also for brain health early in adolescence.

Key words: adolescence; canonical correlation analysis; fitness; lifestyle; multimodal MRI; physical activity

\section{Significance Statement}

An active lifestyle is key for healthy development. In this work, we answer the following question: How do brain neuroimaging markers relate with young adolescents' level of physical activity, fitness, and physical health? Combining advanced wholebrain multimodal MRI metrics with computational approaches, we show a robust relationship between physically active lifestyles and spatially extended, multimodal brain imaging-derived phenotypes. Suggesting a wider effect on brain neuroimaging metrics than previously thought, this work underlies the importance of studying physical lifestyle, as well as other brain-body relationships in an effort to foster brain health at this crucial stage in development.

Received May 4, 2020; revised Sep. 10, 2020; accepted 0ct. 10, 2020.

Author contributions: P.S., T.W., H.D., and H.J.-B. designed research; P.S., T.W., C.W., and N.B. performed research; P.S., T.W., and C.W. analyzed data; P.S. wrote the first draft of the paper; P.S. and H.J.-B. wrote the paper; T.W., C.W., N.B., M.C., D.P., M.B., S.F., E.D., J.A., A.M.W., G.D., T.E.N., S.S., H.D., and H.J.-B. edited the paper.

Fit to Study was supported by the Education Endowment Foundation and Wellcome Trust Education and Neuroscience Program Grant 2681. H.J.-B. was supported by Wellcome Trust 110027/Z/15/Z and Oxford National Institute for Health Research Biomedical Research Center. G.D. was supported by the United Kingdom Medical Research Council MR/K006673/1. T.E.N. was supported by Wellcome Trust 100309/Z/12/Z. The Wellcome Center for Integrative Neuroimaging was supported by core funding from Wellcome Trust 203139/ Z/16/Z. We thank all the Fit to Study investigators (https://www.fit-to-study.org/investigators) for contributions to the trial; Emma Eldridge, Emily Plester, Emily Curtis, Andy Meaney, Patrick Esser, Johnny
Collett, Thomas Smejka, Jack Possee, Oliver Bushnell, Eneid Leika, and Cyrus Goodger for help with data collection; and all of the pupils, and their parents, who took part in, and fully engaged with, each aspect of this brain imaging substudy.

The authors declare no competing financial interests.

Correspondence should be addressed to Piergiorgio Salvan at piergiorgio.salvan@ndcn.ox.ac.uk.

https://doi.org/10.1523/JNEUROSCI.1260-20.2020

Copyright $\odot 2021$ Salvan et al.

This is an open-access article distributed under the terms of the Creative Commons Attribution License Creative Commons Attribution 4.0 International, which permits unrestricted use, distribution and reproduction in any medium provided that the original work is properly attributed. 


\section{Introduction}

The World Health Organization encourages early positive lifestyle choices aimed to improve both physical and mental health (World Health Organization, 2010). Physical activity is a powerful and rapid means to improve fitness and physical health throughout the life-span (Cotman, 2002; Hillman et al., 2008). During adolescence, however, levels of physical activity decline (Guthold et al., 2020).

Public health guidelines recommend that school-aged children engage in $60 \mathrm{~min}$ of moderate-to-vigorous physical activity daily (Piercy and Troiano, 2018), yet globally only $\sim 22 \%$ of boys and $15 \%$ of girls achieve that (Guthold et al., 2020). In addition to its importance to physical health, there is growing evidence that a physically active lifestyle during childhood is associated with improved mental and cognitive health through adulthood (Department of Health \& Human Services Office of Disease Prevention and Health Promotion, 2000). While there is limited available evidence in adolescents, similar patterns have been reported (Lubans et al., 2016).

A body of work has studied the relationship between single physical measures of activity, fitness, or body mass, and separate MRI metrics of brain structure, microstructure, or function, showing focal neural correlates (for review, see Donnelly et al., 2016; Valkenborghs et al., 2019). However, it is unlikely that a single physical measure fully captures active lifestyles, or that a single MRI metric fully quantifies the condition of the brain. Rather, lifestyles are better characterized by a range of physical measures, and the state of the brain is better quantified by combinations of metrics.

Multimodal MRI can probe different aspects of brain structure and function. While each metric provides an indirect probe of the underlying biology, in combination they provide insights into a range of biological processes (Tardif et al., 2016). Further, these measures can be acquired simultaneously across the whole brain. Many previous brain imaging studies of physical activity and fitness have focused on the hippocampus, where changes in noninvasive imaging measures of tissue volume or perfusion have been argued to relate to processes of neurogenesis and angiogenesis triggered by exercise (van Praag et al., 1999; Pereira et al., 2007; Chaddock et al., 2011; Thomas et al., 2012). However, in addition to such focal changes, more global biological processes might also be triggered by exercise (Tardif et al., 2016). It remains unknown whether whole-brain patterns of multimodal brain metrics are related to cardiorespiratory fitness, physical activity, and physical health.

Physical activity influences physical health and contributes to physical fitness, but both activity and fitness may be considered part of an underlying, latent factor. In order to characterize a phenotype of physical lifestyle, measuring whole-day physical activity levels during a normal school week is therefore at least as important as assessing gold-standard measures of cardiorespiratory fitness, such as $\mathrm{VO}_{2} \max$ measured on an incremental steptest on a cycle ergometer.

In this study, in 50 12-year-old pupils, we acquired multimodal whole-brain MRI metrics to measure resting state networks (RSNs), gray matter (GM) volume and perfusion, cortical surface (area and thickness), white matter (WM) microstructure, and myelin content (R1 and R2*), resulting in a total of 18 different metrics. These metrics are combined into multimodal wholebrain phenotypes whose variation across individuals can be interrogated. We also acquired a rich set of variables depicting physical lifestyle, measuring cardiorespiratory fitness $\left(\mathrm{VO}_{2} \mathrm{max}\right.$ and workload), objective physical activity (7 $\mathrm{d}$ actigraphy, measuring total
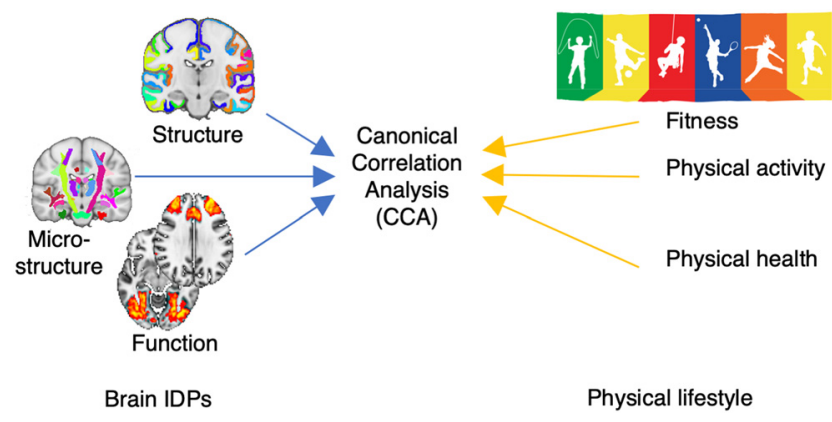

Figure 1. Summary of statistical analysis. In order to test the individual covariation between brain IDPs and physical measures of fitness, physical activity and physical health, we aimed to identify one single mode of covariation using CCA, while taking into account the hierarchical structure represented by schools.

week time of brief bursts and long-lasting physical activity), and reported (questionnaire item) and physical health (resting heart rate, blood pressure, and body mass index) (Fig. 1). We hypothesized that, across pupils, intersubject differences in brain phenotypes covaried with differences in physical lifestyle, independent of sex, socioeconomic status, age, pubertal level, and school. A single holistic multivariate analysis allowed us to identify a latent mode of covariation between brain and physical phenotypes, representing a pattern of active physical lifestyle features that significantly covaries with spatially extended patterns of brain metrics.

\section{Materials and Methods}

\section{Participants and recruitment}

Year 7 pupils from a subset of 10 United Kingdom schools participating in the Fit to Study project Main trial (total 93 schools) were invited to take part in a brain imaging substudy (Wassenaar et al., 2019). The 10 recruitment schools were selected for being conveniently located for travel to Oxford. Researchers visited recruitment schools to pitch the study to pupils and collected pupils' expressions of interest. For each school, an upper limit was determined in order not to over-recruit from a given school. For schools in which the number of interested pupils exceeded this limit, pupils with lower physical activity scores (based on values already collected through the Main trial) were given priority to select a sample that was the most representative as possible of the entire population. The expressions of interest, however, varied greatly by school. Some schools were therefore topped up with more pupils despite creating an imbalance to pragmatically increase study sample size.

After taking consent and assent in accordance with the University of Oxford ethical guidelines (CUREC reference number: R51313/RE001), 61 pupils were recruited to the brain imaging substudy. Participants attended a testing session at the University of Oxford during which brain imaging, cognitive, and behavioral data were collected.

The analysis required high-quality complete multimodal MRI data. One participant withdrew during the scan session. One complete dataset was lost because of hardware failure. Of the remaining datasets, quality control process identified issues with data quality (e.g., head motion, ringing artifacts, blurring, etc.) in one or more modalities in 9 pupils. Therefore, only 50 pupils (median age: 12 years; 26 females, $52 \%$; Table 1) had high-quality, complete multimodal MRI data that could be taken forward into our final analysis. This substudy population is representative of the larger Fit to Study population in terms of demographics (Table 1). However, the substudy pupils were more active and less likely to qualify for free school meals. These differences can be explained by difficulties in recruitment (i.e., low socioeconomic status households were less keen to travel and more active pupils might have been more interested in participation). All statistical analyses were conducted on this sample of 50 pupils sampled from 10 schools (Table 2). The number of participants per school ranged from 1 to 13 . We consistently followed the recruitment process described above, but levels of interest varied 
Table 1. Demographics, socioeconomic status, and self-reported physical activity $^{\mathrm{a}}$

\begin{tabular}{lrl}
\hline & MRI substudy $(n=50)$ & Main trial $(n=15,956)$ \\
\hline Demographics & & \\
Age, mean (SD), years & $12(0.27)$ & $12.5(0.294)$ \\
Female, no (\%), yes & $26(52 \%)$ & \multicolumn{1}{l}{$8931(56.0 \%)$} \\
$\quad$ Pubertal development level, mean (SD) & $2.216(0.59)$ & - \\
Socioeconomic status & & \\
$\quad$ IMD, mean (SD) & $7.82(1.96)$ & \multicolumn{2}{l}{$2664(16.7 \%)$} \\
$\quad$ FSM, no (\%), yes & $1(2 \%)$ & \\
Self-reported physical activity & & $4.42(1.92)$ \\
$\quad$ Reported active days, mean (SD) & $5.43(1.83)$ & \\
\hline
\end{tabular}

${ }^{a} \mathrm{MD}$, Index of multiple deprivation; FSM, free school meal.

Table 2. Sampling frequency by school

\begin{tabular}{lcr}
\hline School & No. of pupils & $\%$ \\
\hline S-1 & 1 & 2 \\
S-2 & 2 & 4 \\
S-3 & 10 & 20 \\
S-4 & 6 & 12 \\
S-5 & 8 & 16 \\
S-6 & 1 & 2 \\
S-7 & 1 & 2 \\
S-8 & 1 & 2 \\
S-9 & 13 & 26 \\
S-10 & 7 & 14 \\
\hline
\end{tabular}

${ }^{a}$ Values in the first column School are arbitrary.

considerably across schools because of several reasons, including variable interest from pupils, concerns from parents regarding the study or travel distance, and unavailability during summer holidays.

\section{Behavioral testing}

All pupils underwent a half-day testing session at the Functional Magnetic Resonance Imaging of the Brain (FMRIB) building in Oxford during summer 2017. Over a period of $\sim 5 \mathrm{~h}$, and with multiple breaks, pupils performed, in this order, cognitive testing, multimodal MRI scans, physical health monitoring, and physical activity testing. Actigraphy monitoring aimed at capturing activity levels over a normal school week time was conducted previous to the day of testing at FMRIB.

\section{Cardiorespiratory fitness}

Objective measures of cardiorespiratory fitness were acquired through an incremental step-test on a cycle ergometer (Lode Excalibur Sport). We then extracted values for maximal oxygen consumption per kilogram $\left(\mathrm{VO}_{2} / \mathrm{kg} \max \right)(\mathrm{ml} / \mathrm{min} / \mathrm{kg})$, and work load maximum (Watts) as primary measures of interest.

\section{Physical activity}

Objective physical activity was assessed over 5 weekdays and 2 weekend days using the Axivity AX3 wrist-worn accelerometer (Open Lab, Newcastle University) (Ladha et al., 2013). We therefore chose to define a valid wear day as 12 consecutive hours from 08:00 to 20:00 to capture travel to and from school and after-school sports and activities. To account for later weekend waking times, we accepted any consecutive $10 \mathrm{~h}$ period between 08:00 and 20:00 on Saturdays and Sundays, and standardized total activity to $12 \mathrm{~h}$. We then aimed to capture both brief bursts and long-lasting activity. We summarized raw accelerometer data from three axes of movement into the signal vector magnitude, or activity "count," expressed per $60 \mathrm{~s}$ epoch and also per $1 \mathrm{~s}$ epoch to characterize sustained bouts of activity and also shorter bursts of movement. Axivity's Open Movement GUI software calculated whether each $60 \mathrm{~s}$ epoch was spent in sedentary, light, moderate, or vigorous activity by applying "cut-points" or "count" thresholds corresponding to different activity intensities derived from a validation study with young people aged 8-14 (Phillips et al., 2013). The software identified nonwear time as periods of at least 30 consecutive minutes of zero activity counts. We used a bespoke program, designed to handle large volumes of data, to apply the same cut-points to each $1 \mathrm{~s}$ epoch. Participants who had at least three valid weekdays and one valid weekend day were included in the analysis (Troiano et al., 2014). For both brief bursts and long-lasting physical activity, participants' total minutes of sedentary, moderate, and vigorous activity per day were calculated.

\section{Physical health}

Physical health was assessed on the day of testing at rest (prior cardiorespiratory testing) by measuring heart rate and systolic and diastolic blood pressure. Compared with publicly available age-matched normative values (Flynn JT et al., 2018), blood pressure (5th-95th percentiles) was found within healthy values (normative values for 12 years old pupils: systolic: $102-131$ with average of 113 ; diastolic: $61-83$ with average of 75 ; study sample, systolic: median $=106,5$ th-95th percentiles: $89-123$; diastolic: median $=73$, fifth-95th percentiles: $58-85$ ).

\section{Negative behaviors not considered in the analysis}

As part of the study, we also obtained ethics to ask pupils information about negative behaviors, such as smoking, drinking alcohol, or drug use. However, none of the pupils reported having used any of these substances.

\section{MR imaging}

MRI acquisition parameters

All MRI scans were conducted during summer 2017 at the Oxford Center for FMRIB using a 3T Siemens Magnetom Prisma scanner with a 32-channel head coil.

The MRI protocol included the following:

T1 weighted (T1w) three-dimensional rapid gradient echo sequence (3D MPRAGE): $\mathrm{TR}=1900 \mathrm{~ms} ; \mathrm{TE}=3.97 \mathrm{~ms}$; flip angle $=8^{\circ}$; FOV $=192$ $\mathrm{mm}$; voxel size: $1 \times 1 \times 1 \mathrm{~mm}$. Sequence duration: $5 \mathrm{~min} 31 \mathrm{~s}$.

1. Resting-state functional MRI (rs-fMRI): multiband EPI sequence; $\mathrm{TR}=933 \mathrm{~ms} ; \mathrm{TE}=33.40 \mathrm{~ms} ; \mathrm{FOV}=192 \mathrm{~mm} ; 72$ slices; voxel size: $2 \times 2 \times 2 \mathrm{~mm}$; multiband acceleration factor $=6$. Sequence duration: $10 \mathrm{~min} 10 \mathrm{~s}$. For each scan, 644 volumes were acquired. Participants were asked to look at a fixation cross, blink normally, try not to fall asleep, and try not to think about anything in particular. A field map was also acquired to correct for inhomogeneity distortions. Sequence duration: 1 min $34 \mathrm{~s}$.

2. Diffusion-weighted MRI (DW-MRI): multishell, multiband EPI sequence; $b$ values $=0,1250,2500 \mathrm{~s} / \mathrm{mm}^{2}$, with, respectively 11,60 , 60 diffusion-weighted directions; $\mathrm{TR}=2483 \mathrm{~ms}$; $\mathrm{TE}=78.20 \mathrm{~ms}$; FOV $=214 \mathrm{~mm}$; voxel size: $1.75 \times 1.75 \times 1.75 \mathrm{~mm}$; multiband acceleration factor $=4$. Sequence duration: $5 \mathrm{~min} 40 \mathrm{~s}$. In addition, $4 \mathrm{~b}=$ $0 \mathrm{~s} / \mathrm{mm}^{2}$ images were acquired with reversed phase encoding, for the purpose of EPI distortion correction. Sequence duration: $32 \mathrm{~s}$.

3. Quantitative FLASH-MRI (Weiskopf et al., 2013): two 3D multiecho FLASH datasets, one predominantly proton-density weighted (PDw, flip angle $=6 \mathrm{deg}$ ), and one predominantly T1w (flip angle $=21$ deg); FOV = $256 \mathrm{~mm}$; voxel size: $1 \times 1 \times 1 \mathrm{~mm}$; TR = $25 \mathrm{~ms}$; first $\mathrm{TE}=2.34 \mathrm{~ms}$; eight equally space echoes, echo spacing $=2.3$; GRAPPA acceleration factor $=2$ in both phase-encoded directions, with 40 reference lines in each direction. Duration for each FLASH sequence: $5 \mathrm{~min} 11 \mathrm{~s}$. Two single-echo, low-resolution (4 mm isotropic) FLASH scans were acquired before each high-resolution scan; identical FOV; TR $=4 \mathrm{~ms}$; TE $=2 \mathrm{~ms}$; one was acquired receiving on the 32-channel receive head coil, the other receiving on the body coil. To correct for the effect of RF inhomogeneities, the local RF field was mapped using a 2D DAM method with a FLASH readout.

4. Pseudo-continuous arterial spin labeling with background presaturation (Okell et al., 2013): six imaging blocks, each with different post-labeling delays: $0.25,0.5,0.75,1,1.25$, and $1.5 \mathrm{~s}$. Arterial blood was magnetically tagged using a labeling duration of $1.4 \mathrm{~s}$. Other imaging parameters were as follows: single-shot EPI; TR $=4100 \mathrm{~ms}$; 
$\mathrm{TE}=14 \mathrm{~ms} ; \mathrm{FOV}=220 \mathrm{~mm}$; voxel size: $3.4 \times 3.4 \times 4.5 \mathrm{~mm}$. Sequence duration: $5 \min 34 \mathrm{~s}$.

In order to provide a more comfortable experience, during all structural scans, a wildlife documentary was shown. A fixation cross was instead shown during rs-fMRI and ASL in order not to bias cognitive processing to certain areas/networks during assessment of resting brain activity.

\section{MRI preprocessing}

MRI data were processed primarily using FSL software (Jenkinson et al., 2012) and FreeSurfer (Dale et al., 1999).

Gradient distortion correction (GDC). GDC was applied within image analysis pipelines using tools developed by FreeSurfer and HCP groups (https://github.com/Washington-University/Pipelines), using the Siemens scanner-specific table of gradient nonlinearities.

Structural. Brain extraction was performed in native space after GDC unwarping using FSL BET (Smith, 2002). Tissue-type segmentation was estimated based on FSL FAST (Zhang et al., 2001), providing hard segmentation as well as partial-volume images for each tissue type. This tool was also used to provide a fully bias-field-corrected version of brain extracted structural brain images. Subcortical structures were modeled using FSL FIRST (Patenaude et al., 2011).

Cortical surface reconstruction. Subject-specific cortical surface reconstruction and cortical parcellation were estimated based on the GDC, brain-extracted T1 image, using the command recon-all from FreeSurfer (Dale et al., 1999).

Registration. Rigid registrations between multimodal MRI native spaces were estimated through FSL FLIRT with boundary-based cost function (Jenkinson et al., 2002; Greve and Fischl, 2009). Nonlinear warps to MNI152 standard-space T1 template were estimated through FSL FNIRT. This set of nonlinear warps is then carried over to all MRI modalities, such as in the case of rs-fMRI.

EPI distortion correction. B0 fieldmap processing was estimated through FSL Topup (Andersson et al., 2003) based on AP-PA image pairs from DWI-MRI protocol.

Functional. rs-fMRI data were preprocessed using a custom pipeline previously validated on developmental datasets (Baxter et al., 2019; Fitzgibbon et al., 2020). rs-fMRI data were corrected for intervolume and intravolume subject head motion and EPI distortions (Andersson and Sotiropoulos, 2015); high pass temporal filtering and GDC unwarping were also applied. Registration to structural was improved by an extra rigid registration step aided by a single-band EPI image. Structured artifacts were removed by FSL ICA+FIX processing (Beckmann and Smith, 2004; Griffanti et al., 2014; Salimi-Khorshidi et al., 2014). The FSL FIX classifier was specifically trained for these data and provided the following scores in leave-one subject-out accuracy: true positive ratio $(\mathrm{TPR})=98.8 \%$; true negative ratio $(\mathrm{TNR})=95.3 \%$; weighted ratio $((3 * \mathrm{TPR}+\mathrm{TNR}) / 4)=97.9 \%$. Independent components (separately identified for each individual) classified as noise (i.e., motion-related, physiological artifacts, MRI acquisition/reconstruction artifacts, etc.), as well as 24 motion confounds, were then regressed into the rs-fMRI signal to obtain denoised (clean) rs-fMRI signal, thus minimizing the effect of head-motion, physiological, and MRI-related artifact at the individual subject level. FSL MELODIC was then used to estimate 50 group-average independent components. We then calculated median absolute (ridge) partial correlation (with a regularization value of 0.1 ) and amplitude for each of the 25 independent components identified as RSNs.

Diffusion. DWI-MRI data were first corrected for eddy currents, EPI distortions, and intervolume and intravolume subject head motion, with outlier-slice replacement, using FSL Eddy (Andersson and Sotiropoulos, 2015). GDP unwarping was then applied (Miller et al., 2016). Diffusion tensor imaging (DTI) fitting was conducted with FSL DTIFIT using a kurtosis model (Behrens et al., 2007). Neurite Orientation Dispersion and Density Imaging (NODDI) modeling was estimated using FSL cuDIMOT based on the Bingham-NODDI model (Tariq et al., 2016). In order to resolve crossing-fiber configurations, multishell voxelwise diffusion was modeled using FSL BedpostX (Jbabdi et al., 2012). Probabilistic tractography was then conducted with FSL ProbtrackX (Behrens et al.,
Table 3. Descriptives of cognitive skills, reported mental health, and reported general health

Mean (SD)

Cognition

Associative task (correct valid answers, \%) 71 (11)

Task switching (switch cost, ms) 548 (333)

Object location task (identification errors, $8 \mathrm{~s}$ delay) 11 (4)

Mental health (Strengths and Difficulties Questionnaire)

Prosocial scale

$8(1.4)$

Hyperactivity scale

$4(2.5)$

Conduct scale

$2(1.5)$

Peer scale

$2(1.9)$

Emotional scale

$3(2.3)$

General Health (Health Behavior in School-aged Children questionnaire)

Life satisfaction

$8(1.6)$

Self-rated health

$4(0.9)$

Multiple health complaints

$13(4.8)$

2007), and 29 major WM bundles were reconstructed as implemented in FSL AutoPtx (de Groot et al., 2013).

Myelin and iron maps. Quantitative MRI data were processed to produce the quantitative maps of myelination (1/T1) and iron level $(1 / \mathrm{T} 2 *)$, using the Voxel-Based Quantification toolbox (Callaghan et al., 2014) in Statistical Parametric Mapping (http://www.fil.ion.ucl.ac.uk/spm/). Although $\mathrm{R} 1$ (1/T1, longitudinal relaxation rate) and $\mathrm{R} 2 *(1 / \mathrm{T} 2 *$, effective transverse relaxation rate) are not direct quantitative maps of myelination or iron (as other biological factors can also affect them), these quantitative maps have a high degree of sensitivity to myelination and iron (Weiskopf et al., 2013; Callaghan et al., 2014; Lutti et al., 2014).

Perfusion. Perfusion images were processed using FSL BASIL (Chappell et al., 2009). Images were first corrected with fieldmap and GDC unwarping; then, to obtain maps of cerebral blood flow and arrival time in absolute units, a calibration step was implemented based on cerebrospinal fluid values.

\section{Image-derived phenotypes (IDPs)}

Each MRI parameter was summarized in a series of IDPs: anatomy-specific average values that span three sets of ROIs. For cortical and subcortical regions, we used the Desikan-Killiany Atlas (84 parcels, 68 cortical, and 16 subcortical) from the individual FreeSurfer parcellation (Fischl et al., 2002). This parcellation was then warped into each (relevant) modality in order not to interpolate MRI-map values. For ASL, we used this parcellation while opting for a conservative approach to minimize coverage issues in frontal and temporal pole ROIs (voxels size being too large for these thin cortical ribbons). We removed bilaterally the frontal and temporal poles ROIs, thus resulting in 80 ROIs for ASL perfusion and 80 ROIs for ASL arrival time (instead of 84 and 84 ROIs). For the white matter, we used the 29 white matter bundles from the AutoPtx reconstruction; first averaged at group level; optimally thresholded; and then warped back to native spaces. For functional activity, 25 group-level RNSs were identified.

A total of 859 IDPs were then fed into statistical analysis. Functional IDPs (RSNs and ASL IDPs) represented 25\% of all IDPs, while WM and GM IDPs represented, respectively, $30 \%$ and $45 \%$ of all IDPs.

\section{Cognitive testing and reported mental health and general health measures}

All measures acquired during the testing are reported in detail in Wassenaar et al. (2019).

\section{Cognitive skills}

Here we considered a summary measure for three tasks of interest: the relational memory task (correct valid answers, \%) (Chaddock et al., 2010); task switching (switch cost, ms) (Hillman et al., 2014); and objectlocation task (identification errors 8 s delay; Table 3) (Pertzov et al., 2012). 
Table 4. MRI modalities and MRI metrics used to define IDPs of brain function and structure ${ }^{\mathrm{a}}$

\begin{tabular}{llll}
\hline MRI modality & Metric & ROI type & No. \\
\hline rs-fMRI & Functional connectivity & RSN & 25 \\
rs-fMRI & Amplitude & RSN & 25 \\
ASL & Perfusion & GM & 80 \\
ASL & Arrival time & GM & 80 \\
T1w-MRI & Volume (VBM-like) & GM & 84 \\
q-MRI & R1 & GM & 84 \\
q-MRI & R2* & GM & 84 \\
T1w-MRI & Surface area & Cortex & 68 \\
T1w-MRI & Surface thickness & Cortex & 68 \\
DW-MRI & DTI - FA & WM & 29 \\
DW-MRI & DTI - MD & WM & 29 \\
DW-MRI & DTI - Kurtosis & WM & 29 \\
q-MRI & R1 & WM & 29 \\
q-MRI & R2* & WM & 29 \\
DW-MRI & NODDI f-iso & WM & 29 \\
DW-MRI & NODDI f-intra & WM & 29 \\
DW-MRI & NODDI DA & WM & 29 \\
DW-MRI & NODDI ODI & WM & 29 \\
\hline
\end{tabular}

${ }^{a}$ Five MRI sequences were used to quantify 18 different MRI metrics. Specific sets of ROls were then used for each MRI metric to extract whole-brain MM IDPs quantifying brain structure, microstructure, function, myelin content, and blood perfusion.

\section{Mental health}

Mental health was assessed with the Strengths and Difficulties Questionnaire (Goodman, 1997).

\section{Questionnaire on general health}

From the Health Behavior in School-aged Children questionnaire (World Health Organization, 2016), we used the positive health items (self-rated health, life satisfaction, multiple health complaints) to measure reported general health.

\section{Experimental design and statistical analyses}

This is a cross-sectional study with a sample size of $N=50$ subjects. Because of the limited sample size compared with the number of variables of interest, we strove to reduce input data and nuisance variables dimensions as much as possible. Standardization of variables before decomposition methods (principal component analysis [PCA] and canonical correlation analysis [CCA]) was applied to avoid variables with disproportionately greater variance driving the decomposition. All statistical analyses were conducted in MATLAB 2018.

\section{Confounds}

Before all statistical analyses, a series of relevant confounds was chosen: age; sex; pubertal developmental level (assessed through the Pubertal Development Rating Scale) (Petersen et al., 1988), a self-report measure of physical development for youths under the age of 16); socioeconomic status (assessed through the United Kingdom Index of Multiple Deprivation); and head size/scaling factor (computed through FSL SIENAX).

On these nuisance variables, we perform a dimensionality reduction through means of PCA $(\mathrm{Nz}=2)$ accounting for $60 \%$ of total variance. These confounds were then regressed out of all IDPs and behavioral variables and the residuals standardized.

Dimensionality reduction of IDPs and physical variables

In order to avoid an overdetermined, rank-deficient CCA solution, and to limit the chances of overfitting, a dimensionality reduction step was performed to both IDPs and physical variables. Using the same approach previously applied by Smith et al. (2015), IDPs were reduced into 10 PCAs $(\mathrm{Nx}=10$; variance explained $=53 \%)$, whereas physical variables were reduced into 5 PCAs $(\mathrm{Ny}=5$; variance explained $=79 \%)$.

\section{CCA}

We sought to characterize a mode of brain-physical covariation across pupils: a data-driven latent factor linking a linear combination of
Table 5. Descriptives of physical variables ${ }^{\mathrm{a}}$

\begin{tabular}{lc}
\hline & Mean (SD) \\
\hline $\mathrm{VO}_{2} \mathrm{max}$, VO \\
$\mathrm{VO}_{2}$ /kg max, workload max (Watts) & $37.2(8.5)$ \\
Vigorous PA, bursts (min per week) & $137.8(25.9)$ \\
Moderate PA, bursts (min per week) & $41.0(34.2)$ \\
Sedentary activities, bursts (min per week) & $247(106)$ \\
Vigorous PA, long-lasting (min per week) & $4075(993)$ \\
Moderate PA, long-lasting (min per week) & $10(14)$ \\
Sedentary activities, long-lasting (min per week) & $320(167)$ \\
Days active during past week & $3502(369)$ \\
Body mass index & $4.9(1.8)$ \\
Resting heart rate (bpm) & $19.5(4.1)$ \\
Blood pressure, systolic (mmHg) & $75(12)$ \\
Blood pressure, diastolic (mmHg) & $106(10)$ \\
\hline
\end{tabular}

${ }^{a}$ Thirteen measures of physical activity, fitness, and physical health were considered in testing the relationship with brain IDPs. Here we report mean (SD) before correcting for demographics and socioeconomic status. PA, Physical activity.

neuroimaging metrics (Table 4) with a linear combination of physical measures (Table 5). To this end, we used CCA, an approach that has successfully been applied in recent studies and that, compared with pairwise association testing, has shown greater sensitivity for complex biological processes and greater explained variance (Smith et al., 2015; Miller et al., 2016).

CCA is a symmetric, cross-decomposition method that characterizes covariation modes between a pair of two-dimensional datasets. This is achieved by finding two sets of free parameters (or canonical coefficients, i.e., one set of coefficient vectors per set of brain metrics and one set of coefficient vectors per set of physical metrics) that maximize the correlation of the projections of the two datasets into the identified latent space (or canonical variates or subject scores). In other words, the variation in mode strength between subjects is maximally correlated. Here, this was computed using MATLAB 'canoncorr' function.

\section{Unbiased statistical inference through block-aware permutation testing}

Deconfounding, as required to ensure that the CCA is not driven by nuisance factors, induces a dependency among the rows of the data submitted to CCA. While this dependency is weak and diminishes with increasing sample size, it represents a violation of the exchangeability assumption required by permutation, which can inflate permutation significance. To account for this deconfounding-induced dependency that violates exchangeability, we use a method that, without changing the canonical correlations, reduces the data from $\mathrm{N}$ observations to $\mathrm{N}-\mathrm{Nz}$ observations that are exchangeable and, thus, can be subjected to a permutation test (Theil, 1965; Winkler et al., 2020). We randomly chose 1000 sets of $\mathrm{Nz}$ rows for removal, conducting 1000 permutations for each set.

Permutations were performed among subjects within school, respecting dependencies given by the hierarchical structure of the data (Winkler et al., 2015). For each of the 1000 repetitions, a $p$ value was computed based on this null distribution for the first CCA mode. Across repetitions, a distribution of statistical significance values was built and the final statistical significance level was computed as its average value. The results of this analysis are shown in Figure $2 b$.

\section{Unbiased estimation of effect size through leave-one school-out} cross-validation

In order to derive an unbiased estimate of the CCA correlation strength that took into account the hierarchical structure in the data, we implemented a leave-one school-out cross-validation (CV) approach. In all but one school, we performed all the above steps (except permutation testing), learning all the coefficients of the standardization steps and of the linear transformations. On the left-out school, we then applied those transformations and predicted left-out pupils' scores in the CCA mode. We repeated this procedure for all folds (here schools). CV performance 

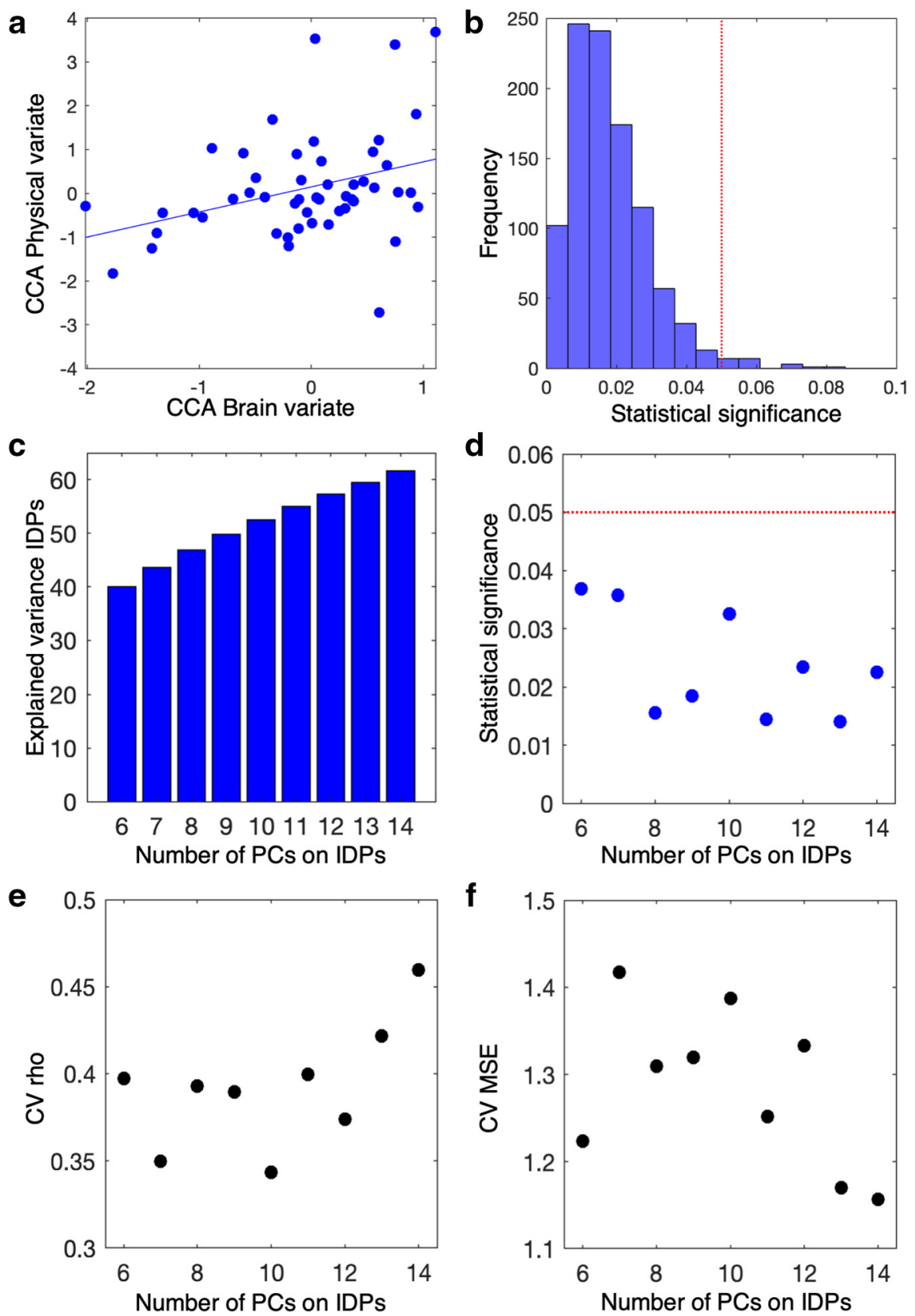

d

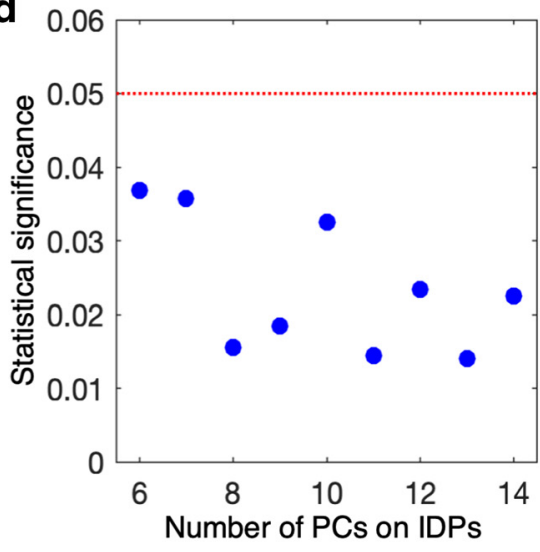

f

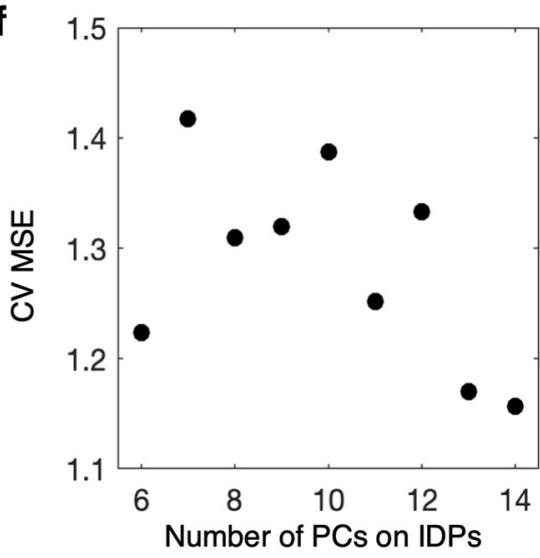

Figure 2. Mode of brain-physical covariation across pupils. The results from CCA highlight one significant mode of brainphysical covariation across pupils. $\boldsymbol{a}$, Scatter plot of cross-validated canonical variates between brain IDP scores and physical scores. Each dot represents a pupil (cross-validated CCA: $\rho=0.34$ ). Statistical significance of CCA was assessed 1000 times, each time comparing the real value against 1000 block-aware permutations taking into account school structure. $\boldsymbol{b}$, Distribution of statistical significance values. The final significance value was assessed as the average of this distribution $(p$ $=0.0130$ ). Red dashed line indicates cutoff of statistical significance of $\alpha=0.05$. c, Explained variance in IDPs as a function of varying the number of $\mathrm{PCs}$ (from $\mathrm{Nx}=6-14$ ). $\boldsymbol{d}$, For each number of $\mathrm{PCS}$, the whole statistical testing pipeline was performed. All analyses led to a statistically significant mode of covariation, showing robustness of identified brain-physical covariation. For each number of PCS, the whole CV pipeline was performed: CV $\rho(\boldsymbol{e})$ and CV MSE $(f)$ across the range of PCS on the IDPs.

was then quantified as the Pearson's $\rho$ correlation coefficient and mean squared error (MSE) calculated between predicted brain and physical canonical covariates (or predicted canonical variates). The results of this analysis are shown in Figure $2 a$.

Supplementary analysis for robustness of identified covariation when varying the number of principal components (PCs) on the IDPs In order to assess whether the identified relationship changes when varying the number of PCs of IDPs, we repeated the whole statistical testing pipeline for a range of PCs numbers (from $\mathrm{Nx}=6$-to- 14 , independently for each PC number) around the previously chosen PCs number $(\mathrm{Nx}=10)$. The results of this analysis are shown in Figure $2 c-f$.

\section{Characterization of brain and physical phenotypes}

We then aimed to characterize the CCA phenotypes: the set of brain measures and the set of physical measures symmetrically linked by the CCA covariance mode. To do this (formally, to characterize the CCA crossed loadings, hereafter referred to as loadings), we follow the procedure described by Smith et al. (2015). On the whole sample, CCA brain loadings were calculated as the pairwise Pearson's partial correlation between CCA physical variate (or subject scores) and the original datasets of brain IDPs, while controlling for the full set of nuisance variables: CCA brain loadings = partial correlation (brain IDPs, CCA physical variate, nuisance variables). The results of this process are shown in Figures 4-6. CCA physical loadings were calculated with the following the same process: CCA physical loadings $=$ partial correlation (physical variables, CCA brain variate, nuisance variables). The results of this process (only for structural IDPs) are shown in Figure 3. CCA loadings are therefore bound between 1 and -1 .

For functional measures, each IDP is represented by a whole-brain RSN. To aid interpretation, for each RSN, its CCA brain loading was multiplied by the group RSN map. The results of this process are shown in Figure $5 a, b$. Then, to derive a summary representation, we concatenated all RSN maps in a $4 \mathrm{D}$ file and computed standardized mean across RSNs, separately for both functional connectivity and amplitude. The results of this process are shown in Figure $5 c, d$.

We then aimed to characterize the average involvement for each type of MRI value. Across IDPs of a MRI metric, we computed the average across CCA brain loadings. This provided a ranked list of MRI parameters representing the average relationship of each MRI metric with pupils' physical scores. The results of this process are shown in Figure 7.

Joint-inferences with univariate measures of cognitive skills, mental health, and general health

The tests for association between the identified CCA mode and the multiple variables measuring the domains of cognitive skills, mental health, and general health were conducted (separately for each domain) using multiple linear regression with nonparametric combination (NPC) implemented in FSL PALM (Winkler et al., 2016). NPC works by combining test statistics or $p$ values of separate (even if not independent) analyses into a single, joint statistic, the significance of which is assessed through synchronized permutations for each of the separate tests. Here we asked whether each CCA covariate (brain covariate while adjusting for physical covariate, and vice versa) was associated with any domain of interest, and the NPC was tested via Fisher statistic with 1000 block-aware permutations while adjusting for nuisance variables in reduced space. For each domain, NPC Fisher significance values were corrected for multiple comparison 


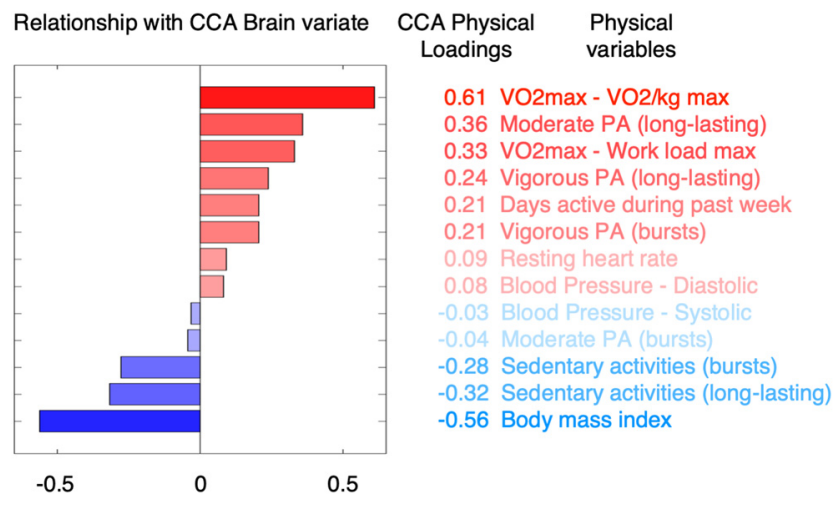

Figure 3. Physical phenotype linked to the brain-physical mode of covariation. Bar plot represents the CCA physical loadings. Each coefficient represents the relationship between each physical metric and subjects' brain IDP scores (or (CA brain variate). Bar plot and variable ranking are matched and color-coded in red/blue in accordance to a positive/negative relationship with the mode of covariation (the magnitude of involvement is further represented through transparency).

testing across CCA covariates (brain and physical) via family-wise error correction (FWE-corr).

\section{Results}

Brain-physical mode of covariation across pupils

Using CCA, we tested the hypothesis that, across pupils, intersubject differences in multimodal whole-brain IDPs covaried significantly with differences in physical lifestyle variables, independent of nuisance variables. We found one significant mode of brain-physical covariation across pupils, linking differences in brain IDPs with individual differences in physical lifestyle (Fig. 2a, CCA: $\rho=0.34, \mathrm{MSE}=1.38$, using leave-one school-out CV; Fig. $2 b, p=0.0130$, significance assessed on 1000 repetitions, each with 1000 block-aware permutations; results remained the same if adjusted for the full set of nuisance variables, $p=0.0312$, significance assessed on 1000 repetitions). We also show that varying the number of PCs on the IDPs (from $\mathrm{Nx}=6$ PCs to 14 PCs) consistently produces the same results (Fig. $2 c-f$ ), showing robustness of the identified relationship across a range of PCs on the IDPs. This mode represents a pattern of brain IDPs that covaries with a pattern of physical variables. We next interrogated this physical phenotype and brain phenotype separately, to determine the patterns that underlie this mode.

\section{Physical phenotype of covariation}

For each physical variable, we calculated the loadings of the physical phenotype relating to the CCA mode (or CCA physical loadings) representing the relationship between each physical variable and the CCA brain variate (or subjects' brain scores) (Fig. 3). We found that pupils who scored higher in the brainphysical mode of covariation were those with higher cardiovascular fitness; those with lower body mass index; those who spent more time doing long-lasting (both moderate and vigorous) physical activity during a normal school week and spent less time being sedentary.

\section{Brain phenotypes of covariation}

In order to interpret brain phenotypes of physical covariation, we calculated the canonical loadings for each IDP of brain structure, microstructure, and function. These loadings (or CCA brain loadings) represent the relationship between each brain IDP and the CCA physical variate (or subjects' physical scores) (to explore the spatial patterns of all structural, microstructural, and functional IDPs, see, respectively, Fig. 4 and Fig. $5 a, b$; for violin plot of CCA brain loadings for all IDPs, see Fig. 6).

We observed that some MRI metrics presented a global and homogeneous involvement in the mode of covariation across ROIs. In order to quantify this tendency, for each MRI metric, we computed the average CCA brain loadings across all ROIs (Fig. 7). We found that the strongest CCA brain loadings were found for GM perfusion (and arrival time) as well as cortical surface area, GM volume, and a number of WM diffusion metrics. MRI metrics with the greatest average CCA brain loadings tended to be characterized by spatially extended and homogeneous involvement across the whole brain. Together, these results show that pupils with greater physical scores were those who also showed global patterns of higher blood perfusion (and lower arrival time, i.e., faster perfusion) in the GM, greater GM volume, greater cortical surface area, greater neurite dispersion anisotropy across WM tracts, as well as greater extraneurite fraction (equivalent to lower intraneurite fraction), and lower neurite orientation dispersion.

We also observed that, although the average CCA brain loadings for RSNs functional connectivity and BOLD amplitude was close to zero, there was great variance across RSNs (Fig. 5). Because RSNs are not binary masks but are instead characterized by spatial distributions, to summarize their pattern of involvement in the mode of covariation, for each voxel we computed the standardized mean CCA brain loadings across RSNs (Fig. $5 c$, d). The resulting maps for functional connectivity (Fig. $5 c$ ) and amplitude (Fig. $5 d$ ) showed both similarities and differences in their patterns of involvement in the mode of covariation. In Figure $5 c$, RSN functional connectivity shows greater positive involvement bilaterally in the parietal cortices, supplementary motor cortex, putamen, and right primary motor cortex, whereas it shows greater negative involvement broadly in the occipital cortices. The peak of positive involvement was localized in the right parietal cortex, whereas the negative involvement was localized in the occipital cortex. In Figure $5 d$, RSN BOLD amplitude shows greater positive involvement in the anterior cingulate gyrus (dACC), superior frontal gyrus, parietal cortices, right inferior frontal gyrus, whereas it shows greater negative involvement broadly in the occipital cortices, and left primary somatosensory cortex. The peak of positive involvement was localized in the dACC, whereas the negative involvement was localized in the occipital cortex. These maps show a common pattern of greater positive involvement bilaterally in the parietal cortices, and a common pattern of negative involvement in the occipital cortices.

\section{Relationship with measures of cognition, mental health, and general health}

We then tested the hypothesis that the identified CCA mode of brain-physical covariation was significantly associated with measures of (1) cognitive skills, (2) mental health, and (3) general reported health. We used a multiple linear regression to test the association between the CCA variates (brain covariate while adjusting for physical covariate, and vice versa) and the outcome measures. Testing an NPC joint-inference for each domain, we found no statistically significant association with cognitive skills (respectively, for brain covariate and physical covariate, NPC Fisher FWE-corr $p=0.2600,0.2360$ ) or general reported health (respectively, for brain covariate and physical covariate, FWE-corr $p=0.7240,0.4910)$. We found a trend toward an association between individual differences in the brain covariate and differences in mental health (NPC Fisher FWE-corr $p=0.0640$ ), whereas no significant association was found for the physical covariate (NPC Fisher FWE-corr $p=0.1830$ ). 
GM Perfusion
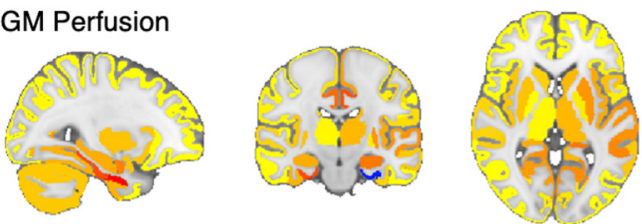

Cortical Surface area
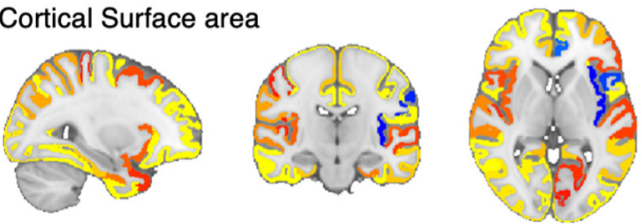

GM Volume
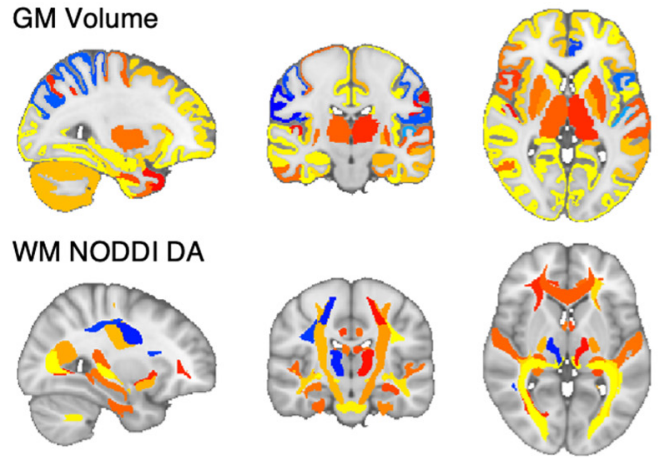

WM DTI MD
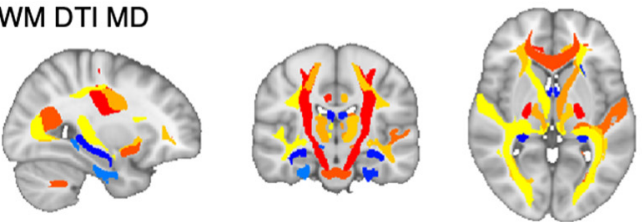

Cortical thickness
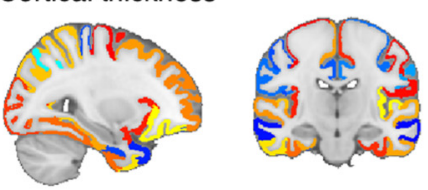

WM DTI FA
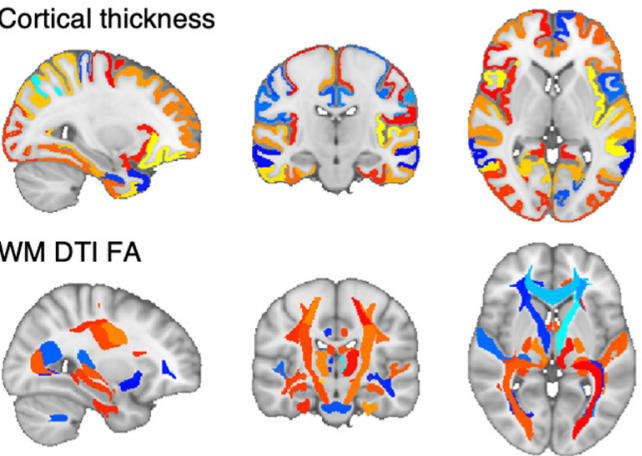

WM QMRI R2*
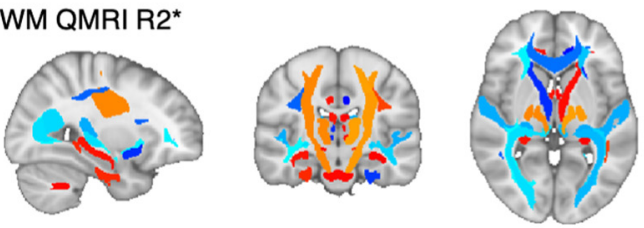
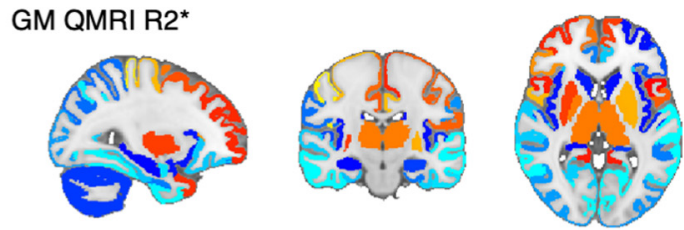

WM QMRI R1
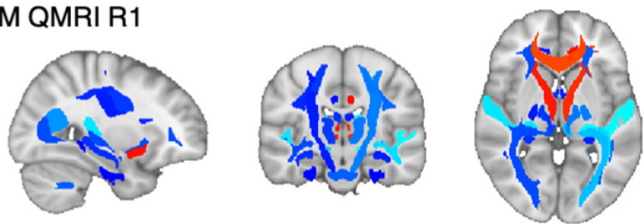

GM QMRI R1
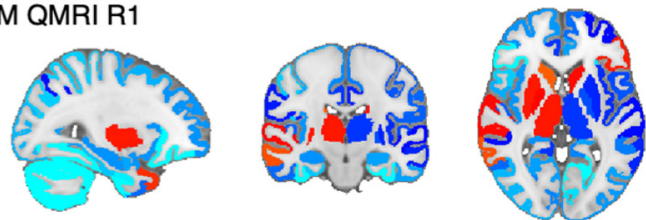

WM NODDI f-iso
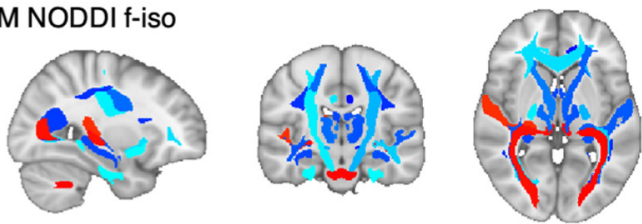

WM NODDI ODI
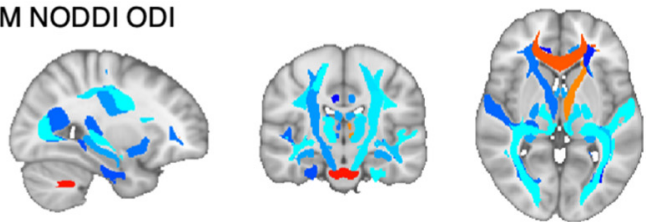

WM DWI Kurtosis
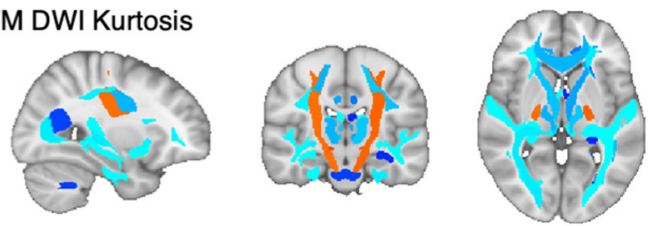

WM NODDI f-intra
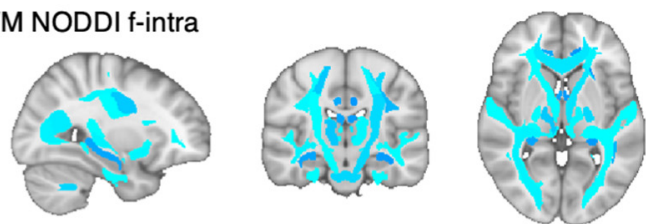

GM Arrival time
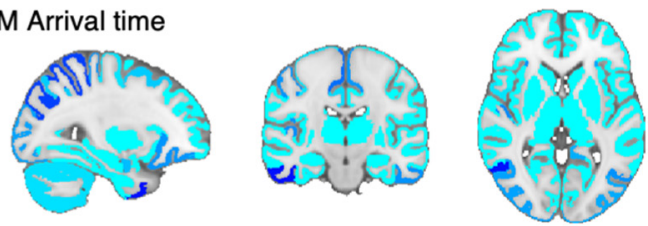

Relationship with

CCA Physical variate

$-0.3$

0

0.3

Figure 4. Structural IDPs and their relationship with the identified phenotype of physically active lifestyle. For each MRI metric and for each structural ROI (thus, for all structural IDPS), the relationship with the identified phenotype of active lifestyle. Hot colors represent a positive relationship with the physical phenotype. Cold colors represent a negative relationship. Structural maps are ranked from top to bottom (left column to right) in accordance to average CCA brain loadings (as shown in Fig. 7).

\section{Discussion}

In this work, we show that, in 12-year-old pupils, physical activity, fitness, and physical health are linked with global patterns of brain structure, microstructure, and function. In this relationship, whole-brain, homogeneous patterns of multimodal brain phenotypes are linked with a specific, latent pattern of physical measures that capture a physically active lifestyle (high fit, high active, low sedentary individuals). This finding hints at the involvement of multiple underlying biological processes and suggests that physical 


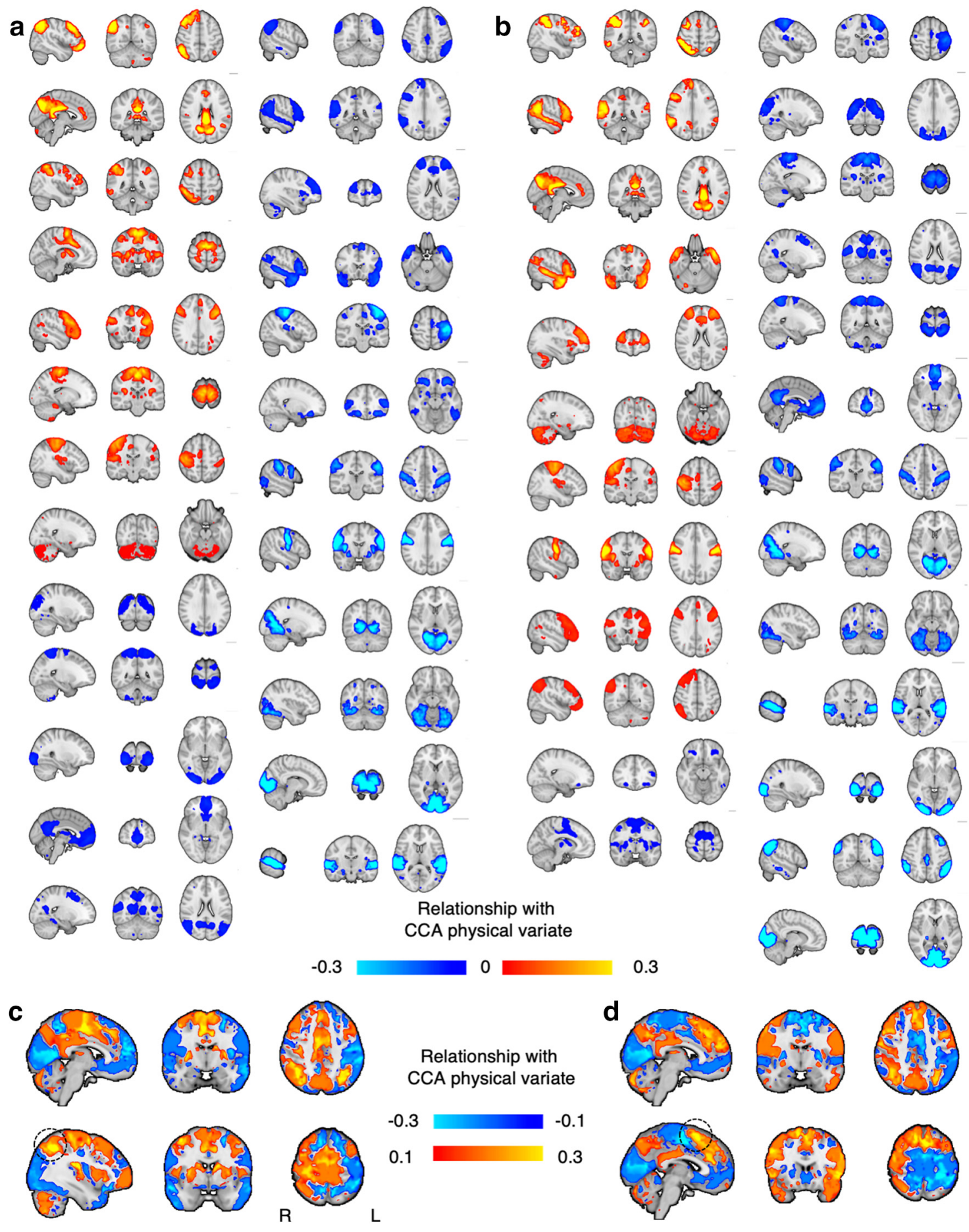

Figure 5. Functional IDPs and their relationship with the identified phenotype of physically active lifestyle. For each RSN, and for both metrics functional connectivity (a) and amplitude (b), the relationship with the identified phenotype of active lifestyle. Hot colors represent a positive relationship with the physical phenotype. Cold colors represent a negative relationship. To aid interpretation, for each RSN, its CCA brain loading was multiplied by the group RSN map. RSNs are here ranked from top to bottom in accordance to their CCA brain loadings. We then concatenated all RSNs maps in a 4D file and computed the mean and SD across RSNs, separately for both functional connectivity and amplitude. c, Standardized mean of CCA brain loadings for RSN functional connectivity. $\boldsymbol{d}$, Standardized mean of CCA brain loadings for RSN amplitude. $\boldsymbol{c}, \boldsymbol{d}$, Top row represents the same brain coordinates. Bottom row represents the respective peak of greater CCA brain loadings. $\boldsymbol{c}, \boldsymbol{d}$, Dashed circle represents the peak value.

health and aerobic exercise might have a wider effect on brain processes than previously thought.

We applied a holistic approach to provide novel insight into the importance of different aspects of a physically active lifestyle in relation to brain structure and function. While high cardiovascular fitness and physical activity are positively linked with the identified brain phenotypes, sedentary activity and body mass index are negatively related. Furthermore, we showed that longlasting physical activity, either moderate or vigorous, is more important to this relationship than brief bursts of activity, suggesting that regular moderate-to-vigorous physical activity might be a better driver to promote brain changes. Together, these findings situate pupils along a latent axis according to their physical phenotype: pupils with high cardiorespiratory fitness 


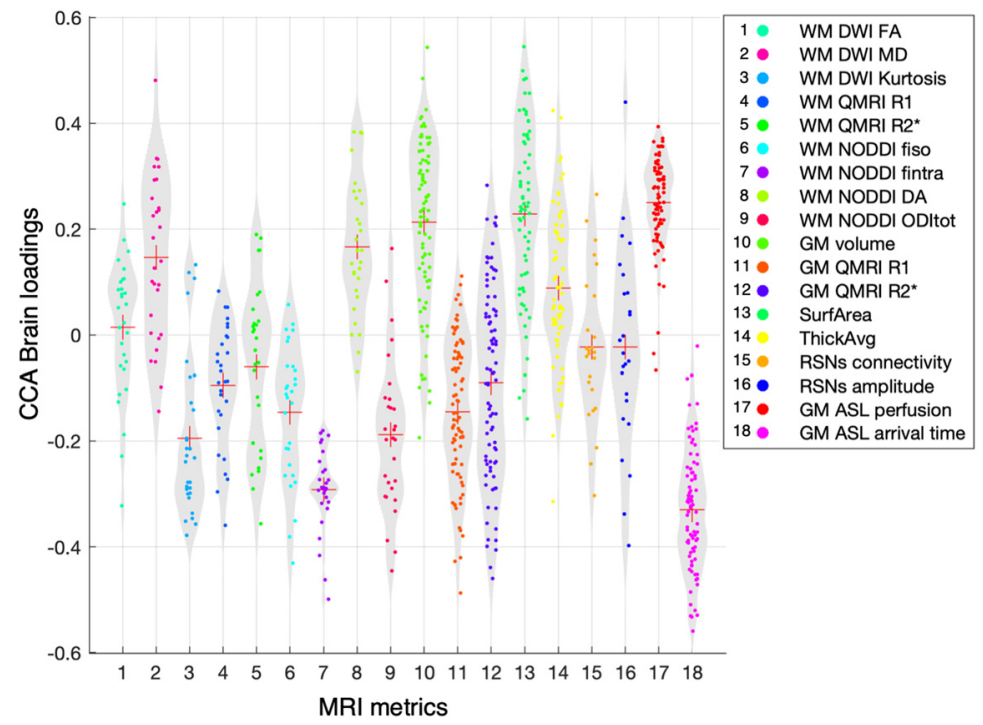

Figure 6. Relationship with physical lifestyle phenotype for all brain IDPs. CCA brain loadings for all 859 brain IDPs divided into each MRI metric. Each dot represents one single IDP.

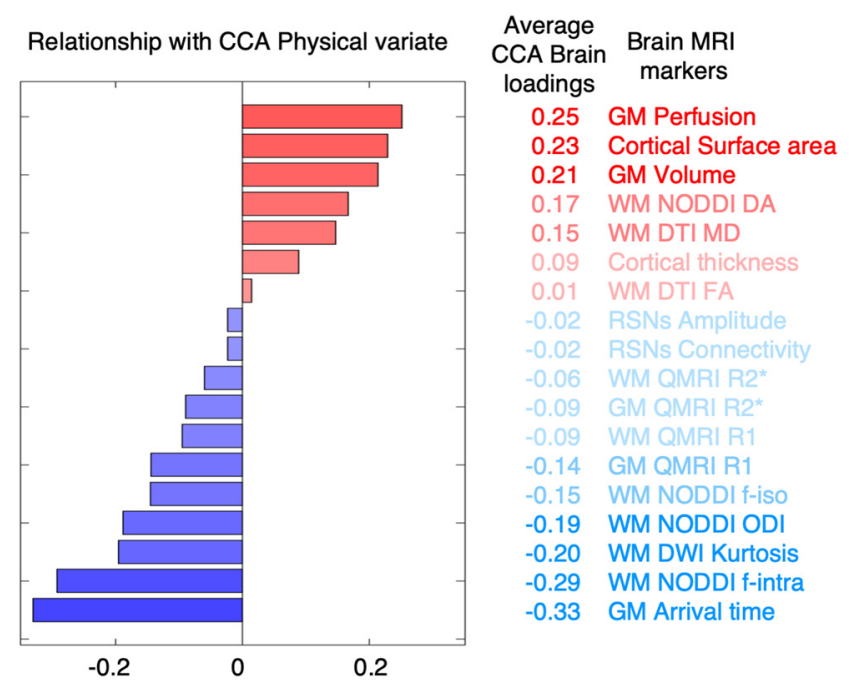

Figure 7. Brain phenotype linked to the brain-physical mode of covariation. Bar plot represents the average CCA brain loadings. Each coefficient represents the relationship between each MRI metric (average across ROls) and pupils' physical lifestyle scores. Bar plot and variable ranking are matched and color-coded in red/blue in accordance to a positive/negative relationship with the mode of covariation (the magnitude of involvement is further represented through transparency).

and performance and with high weekly levels of physical activity, contrast with pupils spending most time in sedentary or low-energy behaviors.

The novelty of this work is the finding of multimodal global brain phenotypes linked with a physically active lifestyle. Although prior work has studied the relationship between single measures of brain structure or function and, separately, physical activity or fitness (Valkenborghs et al., 2019), our approach allowed us to identify latent patterns of multimodal brain IDPs characterized by the involvement of multiple brain regions in the covariation with physical scores. Specifically, greater physical scores were linked with spatially extended patterns of greater blood perfusion and faster arrival time in the GM, greater GM volume, and larger cortical surface area, and in the WM with lower intraneurite density and kurtosis. This result shows that high fitness and physical activity are associated with more global patterns of brain structure than previously thought. Further work is needed to better understand multimodal, spatially extended phenotypes of brain structure (Groves et al., 2012; Douaud et al., 2014). Indeed, it remains unknown how spatially extended brain patterns relate to individual differences in cognition, their level of heritability, as well as to what extent they are susceptible to plasticity. Although it is not possible to infer the presence of a specific biological process or cellular component solely on the basis of MRI measures (Zatorre et al., 2012), these results suggest that high fitness and regular physical activity might have a more widespread impact on brain structure than previously thought.

Previous literature has explicitly focused on studying the effects of aerobic exercise on the hippocampus (Cotman, 2002; Pereira et al., 2007; Chaddock-Heyman et al., 2016; Thomas et al., 2016). Cardiorespiratory fitness is indeed known to promote hippocampal neurogenesis and angiogenesis that, in turn, determines macroscale changes that are also visible via noninvasive neuroimaging (van Praag et al., 1999). Here, we extend the current knowledge beyond a uniquely hippocampal pattern, highlighting the global nature of greater volume and faster perfusion across the whole-brain GM. In other words, variation in hippocampal structure alone does not underlie the brain-physical relationship characterized here. Rather, we observed homogeneous loadings across GM areas. The strongest contributions to our brain phenotype came from perfusion measures. These robust associations found with perfusion metrics are in line with a body of literature showing positive effects of physically active lifestyle on vascular health (Department of Health and Human Services Office of Disease Prevention and Health Promotion, 2000; Vaynman et al., 2004), as well as animal studies linking physical exercise to angiogenesis (Kleim et al., 1996; Rhyu et al., 2010).

Further key insights derive from spatially extended patterns of WM covariation. Although the myelin-sensitive metrics (Quantitative-MRI) in the current study made little contribution to the mode of variation, higher scores on the physical phenotype were associated with lower intraneurite density and kurtosis and, to a lesser extent, with lower neurite orientation dispersion and with greater dispersion anisotropy. It is relevant that the DW-MRI protocol used in this study would be sensitive to diffusion properties within large glial cells, such as astrocytes and oligodendrocytes. Our gradient strength provides sensitivity to length-scales of $\sim 4-6 \mu \mathrm{m}$, with the body size of astrocytes and oligodendrocytes being, respectively, in the order $20 \mu \mathrm{m}$ (Oberheim et al., 2009) and of $14 \mu \mathrm{m}$ (Bakiri et al., 2011), much larger than the average myelinated axon diameter $(<1 \mu \mathrm{m})$ (Liewald et al., 2014). Indeed, astrocytes and oligodendrocytes are the most abundant cells in WM (based on cell counts), accounting for more than half the volume of an MRI voxel (Walhovd et al., 2014). It is thus possible that an increase in size or number of macroglia cells would have a significant effect on the DW-MRI signal, thus contributing to the positive 
association here observed between physical lifestyle scores and WM extraneurite fraction (by construction 1 minus intraneurite density, and specifically, the hindered space outside the neurites prescribed through anisotropic diffusion). Crucially, there exists key histologic evidence from animal studies in support of an increase in astrocyte proliferation and in GFAP levels ( $\mathrm{Li}$ et al., 2005; Uda et al., 2006) and in oligodendrocyte number (Luo et al., 2019) in several areas of the rat brain. Together with this previous literature, the findings here reported may suggest a positive relationship between physically active lifestyle and macroglia cell density across multiple WM tracts, perhaps reflecting a role in providing enhanced metabolic support for neurons. This hypothesis should be tested using imaging alongside more direct measures from ex vivo studies, or using alternative techniques with greater specificity, such as detecting MRS-visible metabolites with greater sensitivity to astrocytes (Brand et al., 1993).

We also report two patterns of RSNs involvement in the mode of brain-physical covariation. We found that a physically active lifestyle was linked with greater connectivity in the parietal cortices and with lower connectivity in the occipital cortices, showing, respectively, increased and decreased BOLD coupling with all RSNs in more active participants. The same phenotype of a physically active lifestyle was also positively related with greater amplitude in local BOLD fluctuations in the dACC and in the parietal cortices, and with lower amplitude in the occipital cortices. Studying both RSNs amplitude (BOLD variance) and functional connectivity (BOLD covariance) can be important to understand possible sources of change and the related neural processes (Garrett et al., 2010; Duff et al., 2018). While greater activity both in functional connectivity and in BOLD amplitude may suggest greater coactivation between the parietal cortices and multiple RSNs across the whole brain, greater BOLD amplitude with no increase in functional connectivity, as observed in the dACC, may suggest greater local activity that results in a decoupling of the dACC from the rest of brain activity. Greater dACC activity during a cognitive control task was previously associated with higher fitness levels in preadolescent children, with greater dACC activity in the high fit group positively related to accuracy in task performance (Voss et al., 2011). In this study, however, we found no significant association between pupil's scores in the mode of brain-physical covariation and differences in cognitive skills. Only a trend for an association with mental health was found, thus not allowing us to infer on the cognitive or mental health relevance of this brain pattern.

Overall, our findings lend support to the growing body of evidence demonstrating a close relationship between the body and the brain. Although the relatively small sample size given the number of variables of interest and the possible cluster effect of schools may represent a limitation of this study, here we used thorough statistical procedures (i.e., block-aware permutation testing and leave-one cluster-out cross-validation) to explicitly deal with this factor, thus producing robust and unbiased statistics. Larger samples might provide power to detect multiple modes of covariation. Also, the results here reported are correlational; therefore, caution is required in interpreting directionality. It is possible that this relationship also represents the other direction of association, and indeed brain structure, microstructure, and function are key drivers of behavioral choices. Nevertheless, our findings suggest that a complex physical phenotype that is influenced by physiology, and lifestyle choices, might have widespread effects on biological processes influencing brain phenotypes. Future studies may test whether improving physical health and fitness through means of activity interventions promotes diffuse neuroplasticity.

In conclusion, this work provides novel insight into the comprehensive relationship between physically active lifestyle and brain structure and physiology in early adolescence. These findings have broad implications for future research, suggesting novel avenues to study the effect of modifiable lifestyle factors as part of wider brain-body relationships. Understanding how physical pathways may foster healthy human brain development can help us to develop better intervention studies aimed at informing public health and education policies.

\section{References}

Andersson JL, Sotiropoulos SN (2015) Non-parametric representation and prediction of single- and multi-shell diffusion-weighted MRI data using Gaussian processes. Neuroimage 122:166-176.

Andersson JL, Skare S, Ashburner J (2003) How to correct susceptibility distortions in spin-echo echo-planar images: application to diffusion tensor imaging. Neuroimage 20:870-888.

Bakiri Y, Káradóttir R, Cossell L, Attwell D (2011) Morphological and electrical properties of oligodendrocytes in the white matter of the corpus callosum and cerebellum. J Physiol 589:559-573.

Baxter L, Fitzgibbon S, Moultrie F, Goksan S, Jenkinson M, Smith S, Andersson J, Duff E, Slater R (2019) Optimising neonatal fMRI data analysis: design and validation of an extended $\mathrm{dHCP}$ preprocessing pipeline to characterise noxious-evoked brain activity in infants. Neuroimage 186:286-300.

Beckmann CF, Smith SM (2004) Probabilistic independent component analysis for functional magnetic resonance imaging. IEEE Trans Med Imaging 23:137-152.

Behrens TE, Berg HJ, Jbabdi S, Rushworth MF, Woolrich MW (2007) Probabilistic diffusion tractography with multiple fibre orientations: what can we gain? Neuroimage 34:144-155.

Brand A, Richter-Landsberg C, Leibfritz D (1993) Multinuclear NMR studies on the energy metabolism of glial and neuronal cells. Dev Neurosci 15:289-298.

Callaghan MF, Freund P, Draganski B, Anderson E, Cappelletti M, Chowdhury R, Diedrichsen J, FitzGerald TH, Smittenaar P, Helms G, Lutti A, Weiskopf N (2014) Widespread age-related differences in the human brain microstructure revealed by quantitative magnetic resonance imaging. Neurobiol Aging 35:1862-1872.

Cassim L, Ladha K, Jackson D, Olivier P (2013) Shaker table validation of OpenMovement AX3 accelerometer. In: Ahmerst (ICAMPAM 2013 AMHERST): In 3rd International Conference on Ambulatory Monitoring of Physical Activity and Movement, pp 69-70.

Chaddock L, Erickson KI, Prakash RS, Kim JS, Voss MW, VanPatter M, Pontifex MB, Raine LB, Konkel A, Hillman CH, Cohen NJ, Kramer AF (2010) A neuroimaging investigation of the association between aerobic fitness, hippocampal volume, and memory performance in preadolescent children. Brain Res 1358:172-183.

Chaddock L, Pontifex MB, Hillman CH, Kramer AF (2011) A review of the relation of aerobic fitness and physical activity to brain structure and function in children. J Int Neuropsychol Soc 17:975-985.

Chaddock-Heyman L, Erickson KI, Chappell MA, Johnson CL, Kienzler C, Knecht A, Drollette ES, Raine LB, Scudder MR, Kao SC, Hillman CH, Kramer AF (2016) Aerobic fitness is associated with greater hippocampal cerebral blood flow in children. Dev Cogn Neurosci 20:52-58.

Chappell MA, Groves AR, Whitcher B, Woolrich MW (2009) Variational Bayesian inference for a nonlinear forward model. IEEE Trans Signal Process 57:223-236.

Cotman C (2002) Exercise: a behavioral intervention to enhance brain health and plasticity. Trends Neurosci 25:295-301.

Dale AM, Fischl B, Sereno MI (1999) Cortical surface-based analysis: I. Segmentation and surface reconstruction. Neuroimage 9:179-194.

de Groot M, Vernooij MW, Klein S, Arfan Ikram M, Vos FM, Smith SM, Niessen WJ, Andersson JL (2013) Improving alignment in tract-based spatial statistics: evaluation and optimization of image registration. Neuroimage 76:400-411. 
Department of Health and Human Services Office of Disease Prevention and Health Promotion (2000) Healthy People 2010: understanding and Improving Health. PsycEXTRA Dataset.

Donnelly JE, Hillman CH, Castelli D, Etnier JL, Lee S, Tomporowski P, Lambourne K, Szabo-Reed AN (2016) Physical activity, fitness, cognitive function, and academic achievement in children: a systematic review. Med Sci Sports Exerc 48:1223-1224.

Douaud G, Groves AR, Tamnes CK, Westlye LT, Duff EP, Engvig A, Walhovd KB, James A, Gass A, Monsch AU, Matthews PM, Fjell AM, Smith SM, Johansen-Berg H (2014) A common brain network links development, aging, and vulnerability to disease. Proc Natl Acad Sci USA 111:17648-17653.

Duff EP, Makin T, Cottaar M, Smith SM, Woolrich MW (2018) Disambiguating brain functional connectivity. Neuroimage 173:540-550.

Fischl B, Salat DH, Busa E, Albert M, Dieterich M, Haselgrove C, van der Kouwe A, Killiany R, Kennedy D, Klaveness S, Montillo A, Makris N, Rosen B, Dale AM (2002) Whole brain segmentation: automated labeling of neuroanatomical structures in the human brain. Neuron 33:341-355.

Fitzgibbon SP, Harrison SJ, Jenkinson M, Baxter L, Robinson EC, Bastiani M, Bozek J, Karolis V, Cordero Grande L, Price AN, Hughes E, Makropoulos A, Passerat-Palmbach J, Schuh A, Gao J, Farahibozorg SR, O'Muircheartaigh J, Ciarrusta J, O'Keeffe C, Brandon J, et al. (2020) The Developing Human Connectome Project (dHCP): automated restingstate functional processing framework for newborn infants. Neuroimage 223:117303.

Flynn JT, Kaelber DC, Baker-Smith CM, Blowey D, Carroll AE, Daniels SR, de Ferranti SD, Dionne JM, Falkner B, Flinn SK, Gidding SS, Goodwin C, Leu MG, Powers ME, Rea C, Samuels J, Simasek M, Thaker VV, Urbina EM (2018) Clinical practice guideline for screening and management of high blood pressure in children and adolescents. Pediatrics 142: e20171904.

Garrett DD, Kovacevic N, McIntosh AR, Grady CL (2010) Blood oxygen level-dependent signal variability is more than just noise. J Neurosci 30:4914-4921.

Goodman R (1997) The Strengths and Difficulties Questionnaire: a research note. J Child Psychol Psychiatry 38:581-586.

Greve DN, Fischl B (2009) Accurate and robust brain image alignment using boundary-based registration. Neuroimage 48:63-72.

Griffanti L, Salimi-Khorshidi G, Beckmann CF, Auerbach EJ, Douaud G, Sexton CE, Zsoldos E, Ebmeier KP, Filippini N, Mackay CE, Moeller S, Xu J, Yacoub E, Baselli G, Ugurbil K, Miller KL, Smith SM (2014) ICAbased artefact removal and accelerated fMRI acquisition for improved resting state network imaging. Neuroimage 95:232-247.

Groves AR, Smith SM, Fjell AM, Tamnes CK, Walhovd KB, Douaud G, Woolrich MW, Westlye LT (2012) Benefits of multi-modal fusion analysis on a large-scale dataset: life-span patterns of inter-subject variability in cortical morphometry and white matter microstructure. Neuroimage 63:365-380.

Guthold R, Stevens GA, Riley LM, Bull FC (2020) Global trends in insufficient physical activity among adolescents: a pooled analysis of 298 population-based surveys with 1.6 million participants. Lancet Child Adolesc Health 4:23-35.

Hillman CH, Erickson KI, Kramer AF (2008) Be smart, exercise your heart: exercise effects on brain and cognition. Nat Rev Neurosci 9:58-65.

Hillman CH, Pontifex MB, Castelli DM, Khan NA, Raine LB, Scudder MR, Drollette ES, Moore RD, Wu CT, Kamijo K (2014) Effects of the FITKids randomized controlled trial on executive control and brain function. Pediatrics 134:e1063-e1071.

Jbabdi S, Sotiropoulos SN, Savio AM, Graña M, Behrens TE (2012) Modelbased analysis of multishell diffusion MR data for tractography: how to get over fitting problems. Magn Reson Med 68:1846-1855.

Jenkinson M, Bannister P, Brady M, Smith S (2002) Improved optimization for the robust and accurate linear registration and motion correction of brain images. Neuroimage 17:825-841.

Jenkinson M, Beckmann CF, Behrens TE, Woolrich MW, Smith SM (2012) FSL. Neuroimage 62:782-790.

Kleim JA, Lussnig E, Schwarz ER, Comery TA, Greenough WT (1996) Synaptogenesis and Fos expression in the motor cortex of the adult rat after motor skill learning. J Neurosci 16:4529-4535.

Li J, Ding YH, Rafols JA, Lai Q, McAllister JP, Ding Y (2005) Increased astrocyte proliferation in rats after running exercise. Neurosci Lett 386:160164.
Liewald D, Miller R, Logothetis N, Wagner HJ, Schüz A (2014) Distribution of axon diameters in cortical white matter: an electron-microscopic study on three human brains and a macaque. Biol Cybern 108:541-557.

Lubans D, Richards J, Hillman C, Faulkner G, Beauchamp M, Nilsson M, Kelly P, Smith J, Raine L, Biddle S (2016) Physical activity for cognitive and mental health in youth: a systematic review of mechanisms. Pediatrics 138:e20161642.

Luo Y, Xiao Q, Wang J, Jiang L, Hu M, Jiang Y, Tang J, Liang X, Qi Y, Dou X, Zhang Y, Huang C, Chen L, Tang Y (2019) Running exercise protects oligodendrocytes in the medial prefrontal cortex in chronic unpredictable stress rat model. Transl Psychiatry 9:322.

Lutti A, Dick F, Sereno MI, Weiskopf N (2014) Using high-resolution quantitative mapping of R1 as an index of cortical myelination. Neuroimage 93:176-188.

Miller KL, Alfaro-Almagro F, Bangerter NK, Thomas DL, Yacoub E, Xu J, Bartsch AJ, Jbabdi S, Sotiropoulos SN, Andersson JL, Griffanti L, Douaud G, Okell TW, Weale P, Dragonu I, Garratt S, Hudson S, Collins R, Jenkinson M, Matthews PM, et al. (2016) Multimodal population brain imaging in the UK Biobank Prospective Epidemiological Study. Nat Neurosci 19:1523-1536

Oberheim NA, Takano T, Han X, He W, Lin JH, Wang F, Xu Q, Wyatt JD, Pilcher W, Ojemann JG, Ransom BR, Goldman SA, Nedergaard M (2009) Uniquely hominid features of adult human astrocytes. J Neurosci 29:3276-3287.

Okell TW, Chappell MA, Kelly ME, Jezzard P (2013) Cerebral blood flow quantification using vessel-encoded arterial spin labeling. J Cereb Blood Flow Metab 33:1716-1724.

Patenaude B, Smith SM, Kennedy DN, Jenkinson M (2011) A Bayesian model of shape and appearance for subcortical brain segmentation. Neuroimage 56:907-922.

Pereira AC, Huddleston DE, Brickman AM, Sosunov AA, Hen R, McKhann GM, Sloan R, Gage FH, Brown TR, Small SA (2007) An in vivo correlate of exercise-induced neurogenesis in the adult dentate gyrus. Proc Natl Acad Sci USA 104:5638-5643.

Pertzov Y, Dong MY, Peich MC, Husain M (2012) Forgetting what was where: the fragility of object-location binding. PLoS One 7:e48214.

Petersen AC, Crockett L, Richards M, Boxer A (1988) A self-report measure of pubertal status: reliability, validity, and initial norms. J Youth Adolesc 17:117-133.

Phillips LR, Parfitt G, Rowlands AV (2013) Calibration of the GENEA accelerometer for assessment of physical activity intensity in children. J Sci Med Sport 16:124-128.

Piercy KL, Troiano RP (2018) Physical activity guidelines for Americans from the US Department of Health and Human Services. Circ Cardiovasc Qual Outcomes 11:e005263.

Rhyu IJ, Bytheway JA, Kohler SJ, Lange H, Lee KJ, Boklewski J, McCormick K, Williams NI, Stanton GB, Greenough WT, Cameron JL (2010) Effects of aerobic exercise training on cognitive function and cortical vascularity in monkeys. Neuroscience 167:1239-1248.

Salimi-Khorshidi G, Douaud G, Beckmann CF, Glasser MF, Griffanti L, Smith SM (2014) Automatic denoising of functional MRI data: combining independent component analysis and hierarchical fusion of classifiers. Neuroimage 90:449-468.

Smith SM (2002) Fast robust automated brain extraction. Hum Brain Mapp 17:143-155.

Smith SM, Nichols TE, Vidaurre D, Winkler AM, Behrens TE, Glasser MF, Ugurbil K, Barch DM, Van Essen DC, Miller KL (2015) A positive-negative mode of population covariation links brain connectivity, demographics and behavior. Nat Neurosci 18:1565-1567.

Tardif CL, Gauthier CJ, Steele CJ, Bazin PL, Schäfer A, Schaefer A, Turner R, Villringer A (2016) Advanced MRI techniques to improve our understanding of experience-induced neuroplasticity. Neuroimage 131:55-72.

Tariq M, Schneider T, Alexander DC, Gandini Wheeler-Kingshott CA, Zhang H (2016) Bingham-NODDI: mapping anisotropic orientation dispersion of neurites using diffusion MRI. Neuroimage 133:207-223.

Theil H (1965) The analysis of disturbances in regression analysis. J Am Statist Assoc 60:1067-1079.

Thomas AG, Dennis A, Bandettini PA, Johansen-Berg H (2012) The effects of aerobic activity on brain structure. Front Psychol 3:86.

Thomas AG, Dennis A, Rawlings NB, Stagg CJ, Matthews L, Morris M, Kolind SH, Foxley S, Jenkinson M, Nichols TE, Dawes H, Bandettini PA, Johansen-Berg H (2016) Multi-modal characterization of rapid anterior 
hippocampal volume increase associated with aerobic exercise. Neuroimage 131:162-170.

Troiano RP, McClain JJ, Brychta RJ, Chen KY (2014) Evolution of accelerometer methods for physical activity research. Br J Sports Med 48:10191023.

Uda M, Ishido M, Kami K, Masuhara M (2006) Effects of chronic treadmill running on neurogenesis in the dentate gyrus of the hippocampus of adult rat. Brain Res 1104:64-72.

Valkenborghs SR, Noetel M, Hillman CH, Nilsson M, Smith JJ, Ortega FB, Lubans DR (2019) The impact of physical activity on brain structure and function in youth: a systematic review. Pediatrics 144:e20184032.

van Praag H, Christie BR, Sejnowski TJ, Gage FH (1999) Running enhances neurogenesis, learning, and long-term potentiation in mice. Proc Natl Acad Sci USA 96:13427-13431.

Vaynman S, Ying Z, Gomez-Pinilla F (2004) Hippocampal BDNF mediates the efficacy of exercise on synaptic plasticity and cognition. Eur J Neurosci 20:2580-2590.

Voss MW, Chaddock L, Kim JS, VanPatter M, Pontifex MB, Raine LB, Cohen NJ, Hillman CH, Kramer AF (2011) Aerobic fitness is associated with greater efficiency of the network underlying cognitive control in preadolescent children. Neuroscience 199:166-176.

Walhovd KB, Johansen-Berg H, Káradóttir RT (2014) Unraveling the secrets of white matter: bridging the gap between cellular, animal and human imaging studies. Neuroscience 276:2-13.

Wassenaar TM, Wheatley CM, Beale N, Salvan P, Meaney A, Possee JB, Atherton KE, Duda JL, Dawes H, Johansen-Berg H (2019) Effects of a programme of vigorous physical activity during secondary school physical education on academic performance, fitness, cognition, mental health and the brain of adolescents (Fit to Study): study protocol for a cluster-randomised trial. Trials 20:189.

Weiskopf N, Suckling J, Williams G, Correia MM, Inkster B, Tait R, Ooi C, Bullmore ET, Lutti A (2013) Quantitative multi-parameter mapping of R1, PD*, MT, and R2* at 3T: a multi-center validation. Front Neurosci 7:95.

Winkler AM, Webster MA, Vidaurre D, Nichols TE, Smith SM (2015) Multilevel block permutation. Neuroimage 123:253-268.

Winkler AM, Ridgway GR, Douaud G, Nichols TE, Smith SM (2016) Faster permutation inference in brain imaging. Neuroimage 141:502-516.

Winkler AM, Renaud O, Smith SM, Nichols TE (2020) Permutation inference for canonical correlation analysis. Neuroimage 220:511706502-516.

World Health Organization (2010) Global recommendations on physical activity for health: WHO Guidelines Approved by the Guidelines Review Committee. Geneva: World Health Organization.

World Health Organization (2016) Growing up unequal: gender and socioeconomic differences in young people's health and well-being: Health Behaviour in School-Aged Children (HBSC) Study. International Report from the 2013/2014 Survey. Geneva: World Health Organization.

Zatorre RJ, Fields RD, Johansen-Berg H (2012) Plasticity in gray and white: neuroimaging changes in brain structure during learning. Nat Neurosci 15:528-536.

Zhang YM, Brady S Smith (2001) Segmentation of brain MR images through a hidden Markov random field model and the expectation-maximization algorithm. IEEE Trans Med Imaging 20:45-57. 